\section{(1)}

CrossMark

\title{
Group 5 drugs for multidrug-resistant tuberculosis: individual patient data meta-analysis
}

\author{
Greg J. Fox ${ }^{1}$, Andrea Benedetti ${ }^{2}$, Helen Cox ${ }^{3}$, Won-Jung Koh ${ }^{4}$, Piret Viiklepp ${ }^{5}$, \\ Shama Ahuja ${ }^{6}$, Geoffrey Pasvol $^{7}$ and Dick Menzies ${ }^{1,8}$ on behalf of the \\ Collaborative Group for Meta-Analysis of Individual Patient Data in MDR-TB ${ }^{9}$
}

\begin{abstract}
Affiliations: ${ }^{1}$ Central Clinical School, University of Sydney, Camperdown, Australia. ${ }^{2}$ Dept of Epidemiology, Biostatistics and Occupational Health, McGill University, Montreal, QC, Canada. ${ }^{3}$ Dept of Medical Microbiology, University of Cape Town, Cape Town, South Africa. ${ }^{4}$ Division of Pulmonary and Critical Care Medicine, Dept of Medicine, Samsung Medical Center, Sungkyunkwan University School of Medicine, Seoul, South Korea. ${ }^{5}$ National Institute for Health Development, Tallinn, Estonia. ${ }^{6}$ Bureau of Tuberculosis Control, New York City Department of Health and Mental Hygiene, Long Island City, NY, USA. ${ }^{7}$ Dept of Life Sciences, Imperial College London, London, UK. ${ }^{8}$ Respiratory Epidemiology and Clinical Research Unit, McGill University, Montreal, QC, Canada. ${ }^{9} \mathrm{~A}$ full list of members of the Collaborative Group for Meta-Analysis of Individual Patient Data in MDR-TB can be found in the Acknowledgements section.
\end{abstract}

Correspondence: Dick Menzies, Room 419, 2155 Guy St, Montreal, QC, H3H 2R9, Canada.

E-mail: Dick.MenziesaMcGill.ca

@ERSpublications

A meta-analysis of patient data found that group 5 drugs have limited benefit in treating patients with MDR-TB http://ow.ly/TIrH304QBci

Cite this article as: Fox GJ, Benedetti A, Cox H, et al. Group 5 drugs for multidrug-resistant tuberculosis: individual patient data meta-analysis. Eur Respir J 2017; 49: 1600993 [https://doi.org/10.1183/ 13993003.00993-2016].

ABSTRACT The role of so-called "group 5" second-line drugs as a part of antibiotic therapy for multidrug-resistant tuberculosis (MDR-TB) is widely debated. We performed an individual patient data meta-analysis to evaluate the effectiveness of several group 5 drugs including amoxicillin/clavulanic acid, thioacetazone, the macrolide antibiotics, linezolid, clofazimine and terizidone for treatment of patients with MDR-TB.

Detailed individual patient data were obtained from 31 published cohort studies of MDR-TB therapy. Pooled treatment outcomes for each group 5 drug were calculated using a random effects meta-analysis. Primary analyses compared treatment success to a combined outcome of failure, relapse or death.

Among 9282 included patients, 2191 received at least one group 5 drug. We found no improvement in treatment success among patients taking clofazimine, amoxicillin/clavulanic acid or macrolide antibiotics, despite applying a number of statistical approaches to control confounding. Thioacetazone was associated with increased treatment success (OR 2.6, 95\% CI 1.1-6.1) when matched controls were selected from studies in which the group 5 drugs were not used at all, although this result was heavily influenced by a single study.

The development of more effective antibiotics to treat drug-resistant TB remains an urgent priority.

This article has supplementary material available from erj.ersjournals.com

Received: May 182016 | Accepted after revision: Oct 012016

Support statement: G.J. Fox was supported by a CJ Martin Post-Doctoral Fellowship (NHMRC App ID 1054107).

Conflict of interest: None declared.

Copyright @ERS 2017 


\section{Introduction}

Multidrug-resistant tuberculosis (MDR-TB) represents a substantial global public health challenge, affecting half a million people each year worldwide [1]. Among the minority of MDR-TB patients who are correctly diagnosed, only $50 \%$ are successfully treated and $16 \%$ die during treatment $[1,2]$. MDR-TB is resistant to the two most effective first-line antibiotics, isoniazid and rifampicin, while extensively drug-resistant (XDR)-TB is also resistant to the most effective second-line drugs (fluoroquinolone antibiotics and second-line injectables (SLIs)). World Health Organization guidelines recommend that at least four effective second-line antituberculosis drugs (as well as pyrazinamide) be combined in the intensive phase of treatment [3]. Second-line drugs are divided into four groups: SLIs, fluoroquinolones, oral bacteriostatic agents ("group 4" drugs) and "group 5" drugs (G5Ds). We considered linezolid, amoxicillin/clavulanate, thioacetazone, clarithromycin, imipenem and terizidone to be G5Ds [2]. These drugs constitute a heterogeneous group of antibiotics with different structures and mechanisms of action [3]. Substantial debate has surrounded the role of these antibiotics as therapy for drug-resistant TB [4], given their potential toxicities and limited evidence of clinical effectiveness for most $[5,6]$. Animal studies suggest that some G5Ds may improve treatment outcomes, when used in combination with standard therapies [7]. Two randomised studies have shown improved treatment outcomes with linezolid in XDR-TB (but not MDR-TB) [8, 9]. A randomised study of clofazimine has been published [10], although the study was not blinded, and the participation rate was not stated, potentially introducing bias. However, recommendations regarding most G5Ds rely upon observational data. While observational studies have shown promising results with some G5Ds, treatment allocation was not randomised in most studies and hence highly prone to selective treatment allocation. Three previously published meta-analyses evaluating the effectiveness of clofazimine $[5,11,12]$ and other G5Ds [5] have been inconclusive. Of these, two were aggregate data meta-analyses $[11,12]$. Such meta-analyses have been unable to account for selection bias and confounding, lacking data about the characteristics of individual patients, which may have affected treatment assignment. In contrast, individual patient data (IPD) meta-analyses are considered the gold standard for combining data from multiple studies [13]. This approach is better able to account for heterogeneity in treatment allocation [14], which is a particularly important issue for analysing regimens such as those used for MDR-TB. Therefore, we aimed to evaluate the effectiveness of G5Ds (treatment success versus death, failure or relapse) using individual patient data from published cohort studies of patients treated for bacteriologically confirmed MDR-TB.

\section{Methods}

\section{Study selection}

Studies were eligible for inclusion if they were included in one of three published aggregate data meta-analyses of MDR-TB treatment (online supplementary table S1) [15-17] and included $\geqslant 25$ patients with MDR-TB. Authors of eligible cohort studies were invited to contribute individual patient datasets. Studies were included if authors agreed to submit the individual patient datasets for their published studies. Aggregate data from studies that did not provide individual patient data were excluded. Other aspects of study selection have been described previously [2]. Of the 32 studies in the original IPD meta-analysis, one was excluded from the present analyses at the request of the authors [18]. The individual patient datasets included participant demographics, clinical markers of disease severity (smear status, cavitation on chest radiograph and bilateral disease), phenotypic drug susceptibility of cultured isolates and detailed medical regimens. Treatment outcomes were defined according to published standard definitions, and included success (comprising cure or treatment completion), death, relapse, treatment failure or loss to follow-up (default) [19].

\section{Individual patient selection}

Patients from the 31 included studies for whom data were available were excluded if they had only extrapulmonary disease but not pulmonary disease or if treatment information was unavailable. Individuals for which details of the antibiotic regimen was unavailable were also excluded.

\section{Definitions}

All included patients had confirmed MDR-TB, with or without resistance to additional antibiotics, as defined by culture and phenotypic drug susceptibility testing [20]. Patients living with HIV infection were included. Primary treatment outcomes (treatment success, failure, death, relapse and loss to follow-up) were defined according to criteria proposed by LASERSON et al. [19].

Exposed patients were those taking one of the G5Ds of interest (amoxicillin/clavulanic acid, clofazimine, linezolid, terizidone, thioacetazone, clarithromycin or imipenem) at any time during their therapy, while unexposed patients were not given that drug. In analyses of the individual drugs, unexposed subjects may have taken another G5D. 
Data were obtained relating to the clinical characteristics of patients, treatment regimens and treatment outcomes. Data were checked for consistency and completeness prior to analysis, using statistical software (Proc Univariate, SAS version 9.3; SAS Institute, Cary, NC, USA; https://support.sas.com/documentation/ 93/index.html).

\section{Descriptive analysis}

Simple descriptive statistics were used to compare the characteristics of patients receiving each G5D, and those not receiving the drug. Pooled treatment outcomes for each G5D and for those patients receiving one or more G5Ds, were calculated using a one-step meta-analysis. Study-level heterogeneity in the univariable analyses was reported using the $\mathrm{I}^{2}$ statistic and its $95 \%$ confidence intervals as described by HigGiNs et al. [21].

\section{Analytic model}

For each G5D that was taken by $\geqslant 40$ individuals, a one-stage meta-analysis was performed using a random effects logistic regression model to evaluate, for each drug, the odds of treatment success (defined as cure or completion) against a combined outcome of treatment failure, relapse and death. A secondary analysis was performed in comparing the odds of treatment success against failure, relapse, death and loss to follow-up (default).

For each G5D the primary comparison was between exposed and unexposed patients in studies where that G5D was available: patients receiving G5Ds (group G5) versus patients not receiving group 5 drugs (group NG5a). In order to account for possible selection bias, a secondary comparison was performed for exposed patients in studies where each G5D was available (group G5) and compared with unexposed patients in studies where that group G5D not available (group NG5b). The latter comparison was used to overcome confounding by indication, since patients in the NG5b group (from a centre where no G5Ds were used) could not be given a G5D regardless of their clinical and treatment characteristics. The rationale for this approach has been described [22].

Unadjusted (crude) and adjusted analyses were performed. Two methods were used to adjust for potential confounding. The rationale for using several alternative analytic methods to account for confounding by indication has been described [22]. First, a traditional hierarchical multivariable logistic regression model was fitted. This aimed to adjust for important baseline characteristics and treatment characteristics likely to influence patient outcomes, including the following covariates: sex, age, disease extent (defined as extensive if the disease was either sputum smear acid-fast bacillus-positive or cavitation was seen on chest radiography), prior treatment with first- or second-line drugs, the number of drugs in the intensive phase and the total duration of antibiotic therapy. These were chosen based upon a directed acyclic graph including possible sources of confounding.

In addition, a second adjustment method used propensity score-based matching to account for differences in baseline characteristics and treatment regimens. This method aimed to address potential confounding by indication, if patients were allocated to treatment on the basis of their clinical characteristics. Application of propensity scores to IPD meta-analysis has been described recently [22]. Propensity scores are an estimate of the probability that a patient with a given set of covariates will be allocated to the exposure (a G5D), and may be used to account for confounding (including confounding by indication) in a regression analysis [23]. Propensity scores were estimated for each individual patient using standard logistic regression, including the following covariates: sex, age, extent of disease, prior TB, prior MDR-TB, number of drugs in the intensive phase and total duration of therapy. Propensity scores were allocated separately according to the selected comparison: 1) within studies where a specific G5D was available (comparing patients taking that G5Ds with those not taking that G5D) or 2) between subjects taking a specific G5D versus subjects in studies where that G5D was unavailable. Each exposed individual was matched with one unexposed individual, where a suitable match could be found. After each selection, unexposed subjects were returned to the pool of subjects and were eligible for repeat selection (i.e. one-to-one matching with replacement). The matching algorithm used nearest-neighbour matching to identify pairs of treated and untreated subjects [24].

The effectiveness of covariate balance achieved by matching was evaluated by calculating the standardised difference of the covariates, and logit of the propensity score, between exposed and unexposed groups [25-27]. Differences between treatment groups of $<10 \%$ for individual covariates implied balance in these potential confounders [28]. Heterogeneity between studies was assessed by calculating $\mathrm{I}_{\mathrm{R}}^{2}$ from the standard error of the random effects and fixed-effects models [29].

Study, treatment and match were random effects in the propensity score models. This provided adjusted odds of treatment success for patients who used G5Ds. Patients were clustered at the level of the study and intercepts and slopes varied across studies. If models did not converge, the covariance requirement between slope and intercept was removed. If convergence still did not occur, then the random intercept 
was removed. If a fixed-effects model was required, this was reported. The models were estimated via penalised quasi-likelihood (Proc Glimmix, SAS 9.3).

Missing covariate values in the individual patient dataset were imputed using multiple imputation (Proc MI, SAS 9.3), propensity scores were estimated and then patients with and without the drug were matched based upon propensity score. Analyses were performed in SAS (9.3).

The study was reported according to the Preferred Reporting Items for Systematic Reviews and Meta-Analyses (PRISMA) guidelines for individual patient data meta-analysis [30]. The authors did not prepare a formal written study protocol. The analytic approach has been described previously [22].

\section{Results}

Individual patient data for 9282 patients was obtained from the authors of 31 studies; in 24 of these studies at least one patient received at least one G5D (figure 1). In these studies, 2191 individuals took regimens containing at least one G5D (table 1). The remaining seven studies where no G5Ds were given included 2875 individuals. 919 patients were given amoxicillin/clavulanic acid, 806 clofazimine, 532 clarithromycin, 705 thioacetazone, 32 terizidone, nine imipenem and 39 linezolid.

Online supplementary table S2 summarises the settings and treatment regimen used in each included study. Online supplementary table S3 shows the number of patients taking G5Ds for each included study. Online supplementary table S4 shows the proportion of non-missing data for each independent variable included in the multivariable regression, grouped according to individual studies. The table also shows the proportion of individuals lost to follow-up in each study. No important issues were identified in checking the individual patient data.

The median (interquartile range) treatment duration for patients taking a G5D was 20.9 (14.6-25.6) months compared to $14.4(6.3-20.0)$ months for those not taking a G5D $(p<0.01)$. 50.8\% of patients taking a G5D had MDR-TB with additional resistance to either fluoroquinolones or injectable antibiotics or both, compared with only $33.1 \%$ of patients not taking a G5D. Most patients taking a G5D were taking only one (67.9\%) or two (26.6\%) G5Ds.

Table 2 shows the pooled treatment outcomes, according to which G5D was taken. When excluding subjects lost to follow-up, 74\% of subjects taking a G5D were treated successfully, compared to $73 \%$ of those not taking a G5D. The proportion with treatment success was similar if patients lost to follow-up were included (61\% versus 58\%).

The proportion lost to follow-up varied considerably; only 8\% (95\% CI 4-11\%) of those taking clofazimine and $6(3-10) \%$ of those taking amoxicillin/clavulanic acid were lost to follow-up, compared to $20 \%$ of those not taking any G5Ds.

Among patients taking clofazimine, patients with smear-positive disease, pre-XDR disease or extrapulmonary TB had reduced treatment success, after adjustment (table 3). Those with an overall longer duration of treatment, or taking at least four effective drugs had an increased odds of success, after adjustment.

Propensity score matching improved the covariate balance between most covariates of individuals with and without G5Ds (table 4). Before matching, standardised difference of the logit of the propensity score was 40.7\% lower among those taking clofazimine compared to those not taking clofazimine, while this was only $0.1 \%$ lower after one-to-one matching with replacement (implying almost perfect covariate balance).

Table 5 shows the relationship between the use of each G5D, where data from $\geqslant 50$ patients were available, and treatment success (compared to death, failure or relapse) using multivariable analysis or propensity score-based matching. When control subjects were obtained only from studies where G5Ds were used (group G5 versus NG5a), adjusted (multivariable) estimates found that treatment success was lower among those taking clofazimine, amoxicillin/clavulanic acid and clarithromycin. After matching, clofazimine, amoxicillin and thioacetazone were no longer associated with reduced treatment success. When controls were selected only from studies where G5D were not used, no benefit was found with use of any G5D.

Table 6 also includes subjects lost to follow-up, comparing treatment success to death, failure relapse and loss to follow-up. Only thioacetazone was associated with increased treatment success (OR 2.6, 95\% CI 1.1-6.1) in the matched analyses when controls were taken from studies where a G5D was not used by any patients (G5D versus NG5b). However, 95\% of the 705 patients treated were thioacetazone were from a single study site (online supplementary table S5) [32]. Heterogeneity in the matched analysis was moderate, with $\mathrm{I}_{\mathrm{R}}^{2}$ estimates of $<40 \%$ seen for most comparisons.

Online supplementary table S6 shows the PRISMA-IPD checklist of items according to be included when reporting an IPD meta-analysis. Individual study quality was not formally assessed. Online supplementary table S7 provides a risk of bias assessment for the included studies. 


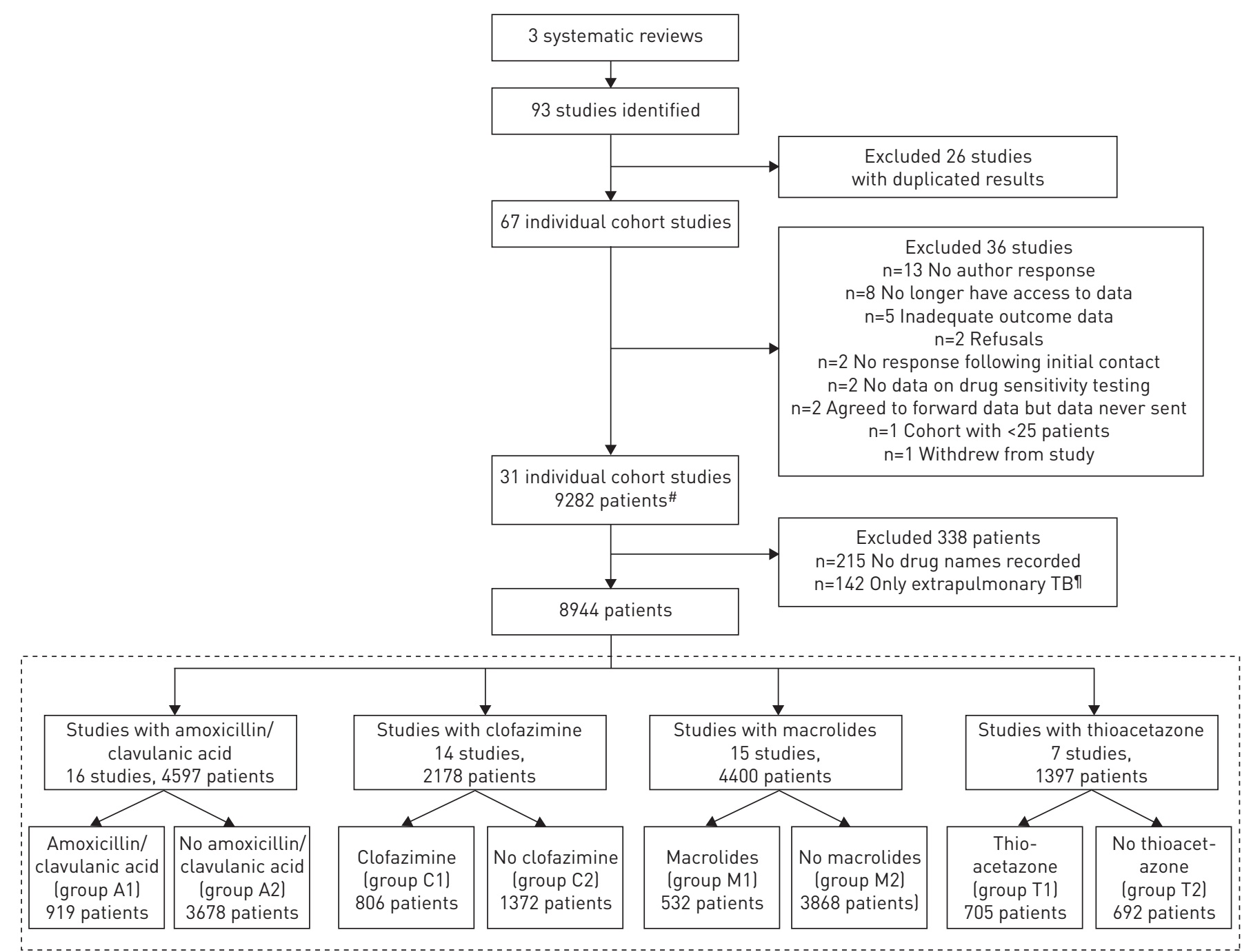

FIGURE 1 Consort diagram for individual patient data meta-analysis of group 5 drugs for multidrug-resistant tuberculosis (TB). Among studies included in the present meta-analyses, 2191 patients from 24 studies took at least one group 5 drug: amoxicillin/clavulanic acid (n=919 patients, 16 studies), clofazimine ( $n=806$ patients, 14 studies), macrolide antibiotics ( $n=532$ patients, 15 studies), thioacetazone ( $n=705$ patients, seven studies), linezolid ( $n=39$ patients, four studies), imipenem ( $n=7$ patients, four studies) and teridizone ( $n=12$ patients, one study). ${ }^{\#}$ : Patients from 31 cohort studies were included in these analyses. The authors of one study included in the previously published meta-analysis withdrew from the present study [2]; ": 19 excluded patients had both extrapulmonary TB and no drug names recorded.

\section{Discussion}

This IPD meta-analysis of 31 studies evaluated the effect of four G5Ds among 2191 patients treated for MDR-TB. We found improvement in treatment outcomes among patients treated with thioacetazone but not among patients who received clofazimine, amoxicillin/clavulanic acid or macrolide antibiotics. Our findings support a conservative approach to using these G5Ds, and suggest that alternative drugs should be used where possible [33].

An interesting finding of this study was improved treatment outcomes among patients taking thioacetazone, when patients in studies taking the drug were compared to patients in studies where the drug was not used. This result predominantly arose from one study from Latvia, which contributed $95 \%$ of the 705 patients who received thioacetazone [32], and achieved a higher treatment success rate among those taking thioacetazone than was seen in other studies. This may reflect, in part, the overall strength of treatment in that setting, where the majority of patients received thioacetazone. Hence, while the finding may reflect a true benefit of the drug, study-specific factors related to patient selection for thioacetazone or differences in the patient populations may also be responsible. The absence of an effect when patients within the same study were used as controls points towards possible confounding by indication, as we have discussed previously [22]. Therefore, this finding should be interpreted with caution. 
TABLE 1 Participant characteristics, classified by whether at least one group 5 drug was used

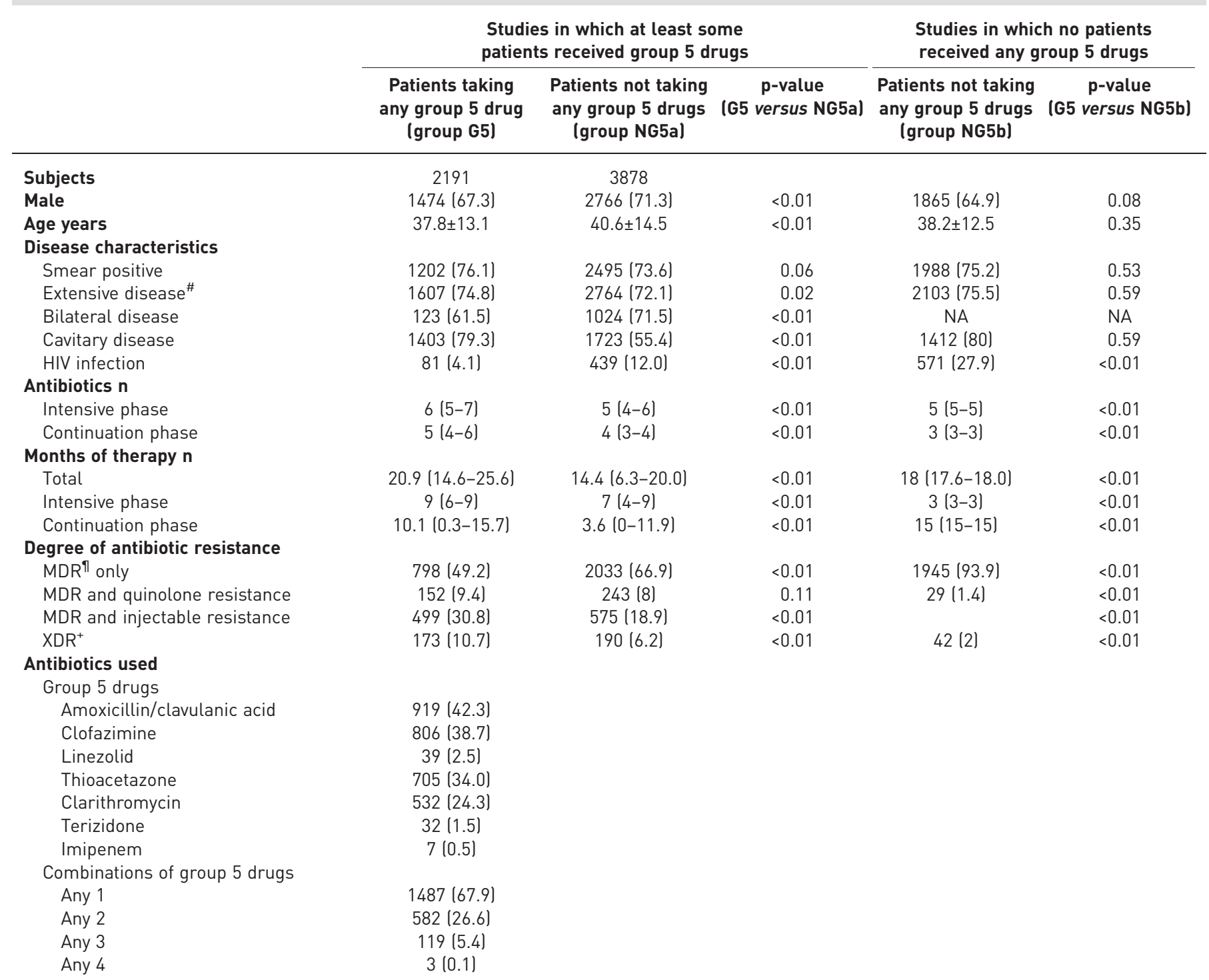

Data are presented as n, $\mathrm{n}(\%)$, mean \pm SD or median (interquartile range), unless otherwise stated. Group 5 drugs are defined as amoxicillin/ clavulanic acid, clofazimine, linezolid, clarithromycin or imipenem. MDR: multidrug-resistant; XDR: extensively drug-resistant; NA: not applicable. ${ }^{\#}$ : defined as a combination of smear positive and cavitary disease; ${ }^{\uparrow}$ : defined as resistant to isoniazid and rifampicin; ${ }^{+}$: defined as MDR plus resistance to an injectable second-line antibiotic and a fluoroquinolone.

We found no benefit with clofazimine using any of our analytic approaches, consistent with a previous meta-analysis including 42 patients taking the drug [5]. This contrasts with a recent randomised controlled trial from China (53 patients taking clofazimine, 52 patients not taking clofazimine) which found a substantial increase in treatment success with clofazimine in MDR-TB [10]. Although the effect estimate was not reported, using the study data we calculated the odds ratio for treatment success with clofazimine in the Chinese study to be 2.4 (95\% CI 1.6-3.2), when compared to those not taking the drug. Importantly, the study was not blinded, and the participation rate was not stated, potentially introducing bias. Another recent cohort study also found improved outcomes with clofazimine for treating XDR-TB [34], and animal studies have shown evidence of faster time to cure using the drug [7]. The explanation for this difference between our meta-analysis and these published studies remains unclear. It is possible that the lack of blinding could have introduced bias in the Chinese study, and confounding by indication may have biased the South African [34] study, although these findings clearly warrant further investigation. In the animal study, use of three active drugs (rather than the usual two) during the continuation phase may have contributed to the greater effectiveness of the clofazimine-containing regimen. Nonetheless, given the observational nature of the cohort studies included in our meta-analysis, 
TABLE 2 Pooled treatment outcomes according to group 5 drug use, among studies where the drug was used

\begin{tabular}{|c|c|c|c|c|c|}
\hline & \multirow[t]{2}{*}{ Events } & \multirow[t]{2}{*}{ Total } & \multirow[t]{2}{*}{ Pooled treatment outcomes ${ }^{\#}$} & \multicolumn{2}{|c|}{ Measures of heterogeneity } \\
\hline & & & & $1^{2}$ & $\tau^{2}$ \\
\hline \multicolumn{6}{|c|}{ Outcomes excluding loss to follow-up } \\
\hline No group 5 drugs & 1835 & 2762 & $75(65-81)$ & 98 (97-98) & 0.059 \\
\hline At least one group 5 drug & 1357 & 1894 & $74(66-81)$ & 94 (92-95) & 0.032 \\
\hline Clarithromycin & 287 & 457 & $74(68-80)$ & $45(0-70)$ & 0.006 \\
\hline Thioacetazone & 491 & 612 & $80(77-83)$ & $0(0-71)$ & 0.000 \\
\hline Terizidone & 13 & 16 & $76(51-100)$ & $0(0-85)$ & 0.000 \\
\hline Linezolid & 12 & 22 & $50(28-71)$ & $0(0-90)$ & 0.000 \\
\hline \multicolumn{6}{|c|}{ Outcomes including loss to follow-up } \\
\hline \multicolumn{6}{|l|}{ Treatment success } \\
\hline No group 5 drugs & 1835 & 3878 & $60(50-70)$ & 98 (97-98) & 0.055 \\
\hline Thioacetazone & 491 & 705 & $70(66-73)$ & $0(0-71)$ & 0.000 \\
\hline Terizidone & 13 & 32 & $30(14-46)$ & $0(0-85)$ & 0.000 \\
\hline Linezolid & 12 & 39 & $28(13-42)$ & $0(0-85)$ & 0.000 \\
\hline \multicolumn{6}{|l|}{ Treatment failure or relapse } \\
\hline No group 5 drugs & 255 & 3878 & $3(2-4)$ & $50(18-69)$ & 0.000 \\
\hline At least one group 5 drug & 253 & 2191 & $9(6-12)$ & $83(75-88)$ & 0.005 \\
\hline Clarithromycin & 97 & 532 & $10(6-14)$ & $49(7-72)$ & 0.004 \\
\hline Clofazimine & 48 & 806 & $2(0-4)$ & $32(0-64)$ & 0.001 \\
\hline Amoxicillin/clavulanic acid & 108 & 919 & $13(6-20)$ & $82(71-88)$ & 0.016 \\
\hline Thioacetazone & 97 & 705 & $14(11-16)$ & $0(0-71)$ & 0.000 \\
\hline Terizidone & 2 & 32 & $20(0-45)$ & $0(0-85)$ & 0.000 \\
\hline Linezolid & 1 & 39 & $33(0-87)$ & $0(0-85)$ & 0.000 \\
\hline \multicolumn{6}{|l|}{ Loss to follow-up } \\
\hline \multicolumn{6}{|l|}{ Death } \\
\hline No group 5 drugs & 672 & 3878 & $11(5-17)$ & 97 (97-98) & 0.021 \\
\hline At least one group 5 drug & 284 & 2191 & $10(6-15)$ & 91 (87-93) & 0.012 \\
\hline Clarithromycin & 73 & 532 & $8(3-13)$ & $75(58-85)$ & 0.007 \\
\hline Clofazimine & 192 & 806 & $14(5-24)$ & $86(77-91)$ & 0.030 \\
\hline Amoxicillin/clavulanic acid & 156 & 919 & $6(2-9)$ & $60(30-77)$ & 0.004 \\
\hline Thioacetazone & 93 & 705 & $13(11-16)$ & $0(0-71)$ & 0.000 \\
\hline Terizidone & 1 & 32 & $6(0-16)$ & $0(0-85)$ & 0.000 \\
\hline Linezolid & 9 & 39 & $25(11-39)$ & $0(0-85)$ & 0.000 \\
\hline
\end{tabular}

Measures of heterogeneity

Data are presented as $\mathrm{n}$ or $\%(95 \% \mathrm{Cl})$, unless otherwise stated. $\tau^{2}$ : the estimated standard deviation of underlying effects across the studies. \#: weighted treatment outcomes [31]; ๆ : defined as cure or treatment completion.

we cannot exclude the possibility that unmeasured confounding resulted in an underestimate of the effect of clofazimine. Hence, our results suggest that additional data from well-designed and -executed randomised trials are still warranted, before widely adopting clofazimine as initial therapy for MDR-TB.

Estimates for the effect of treatment differed depending upon the population from which control subjects were selected. When control subjects (i.e. those not taking a given G5D) were selected from studies where the G5D was available, three drugs (clofazimine, amoxicillin/clavulanic acid and macrolide antibiotics) demonstrated reduced treatment success, but, when controls were drawn from studies where the drug was not available, there was no apparent effect of the drug at all. This finding points toward a potential 
TABLE 3 Univariable analysis of treatment success versus death or treatment failure among patients taking clofazimine

\begin{tabular}{|c|c|c|c|c|c|}
\hline & \multirow[t]{2}{*}{ Subjects $\#$} & \multirow[t]{2}{*}{$\begin{array}{c}\text { Univariable } \\
\text { analysis }\end{array}$} & \multirow[t]{2}{*}{$\begin{array}{c}\text { Multivariable } \\
\text { analysis }\end{array}$} & \multicolumn{2}{|c|}{$\begin{array}{l}\text { Measures of } \\
\text { heterogeneity }\end{array}$} \\
\hline & & & & R statistic & $I_{R}^{2}$ \\
\hline \multicolumn{6}{|l|}{ Subject characteristics } \\
\hline $\mathrm{Age}^{+}$ & 699 & $0.9(0.8-1.0)$ & $0.9(0.8-1.1)$ & 1.038 & 0.037 \\
\hline Male & 699 & $1.1(0.8-1.6)$ & $1.2(0.8-1.7)$ & 1.017 & 0.017 \\
\hline $\begin{array}{l}\text { Smear-positive at diagnosis (versus not } \\
\text { smear-positive) }\end{array}$ & 634 & $0.3(0.2-0.5)$ & $0.3(0.2-0.5)$ & 1.009 & 0.008 \\
\hline Extensive disease $e^{\S}$ & 699 & $0.3(0.2-0.5)$ & $0.3(0.2-0.5)$ & 1.015 & 0.015 \\
\hline Pre-XDR-TB (versus MDR-TB but not XDR-TB) & 699 & $0.5(0.3-0.9)$ & $0.6(0.3-0.9)$ & 1.028 & 0.027 \\
\hline XDR-TB (versus MDR-TB, not pre-XDR-TB) & 322 & $0.5(0.2-1.5)$ & $0.4(0.1-1.2)$ & 1.051 & 0.048 \\
\hline EPTB also present (versus PTB only) & 699 & $0.2(0.1-0.4)$ & $0.2(0.1-0.5)$ & 1.004 & 0.003 \\
\hline \multicolumn{6}{|l|}{ Medical therapy } \\
\hline$<3$ effective drugs & & Ref. & Ref. & & \\
\hline 4 effective drugs (versus <3) & 71 & $2.1(0.2-20.7)$ & $1.0(0.1-13.5)$ & 1.036 & 0.068 \\
\hline 5 effective drugs (versus <3) & 125 & $1.8(0.3-12.4)$ & $1.5(0.2-10.5)$ & 1.025 & 0.048 \\
\hline 6 effective drugs (versus <3) & 197 & $1.7(0.2-12.3)$ & $0.8(0.1-9.6)$ & 1.017 & 0.033 \\
\hline 7 effective drugs (versus <3) & 189 & $0.1(0.0-11.9)$ & $0.3(0.0-6.0)$ & 1.066 & 0.12 \\
\hline 8 effective drugs (versus $<3$ ) & 93 & $2.3(0.3-16.4)$ & $0.1(0.0-1.3)$ & 1.000 & 0.000 \\
\hline \multicolumn{6}{|l|}{ Continuation phase } \\
\hline$<3$ effective drugs & & Ref. & Ref. & & \\
\hline$\geqslant 3$ effective drugs (versus $<3$ ) & 95 & $2.6(0.8-8.5)$ & $2.7(0.7-10.7)$ & 1.020 & 0.040 \\
\hline$\geqslant 4$ effective drugs (versus $<3$ ) & 144 & $2.6(1.0-6.6)$ & $3.2(1.2-8.8)$ & 1.016 & 0.031 \\
\hline$\geqslant 5$ effective drugs (versus $<3$ ) & 368 & $5.0(2.1-11.9)$ & $5.7(2.3-13.8)$ & 1.017 & 0.032 \\
\hline
\end{tabular}

residual confounding by indication, whereby the sicker patients may be systematically offered G5Ds. The use of two alternative control populations, in order to address confounding by indication, is a strength of this study, as described later.

Another intriguing finding was that rates of loss to follow-up varied considerably between patients taking some G5Ds (6\% for amoxicillin/clavulanic acid and 8\% for clofazimine) and patients not given any G5Ds ( $20 \%$ for those not taking a G5D). This could point towards possible ascertainment bias in the group not given G5Ds (e.g. death, failure and relapse may have been underestimated among those lost to follow-up). However, effect estimates were similar regardless of whether participants lost to follow-up were included in the analyses. This suggests that the proportion of individuals lost to follow-up was not an important contributor to treatment outcomes.

A key strength of this study was the inclusion of a large number of patients taking G5Ds across 31 studies. Although the heterogeneity in effect estimates observed between included studies might conceal small effect estimates (i.e. decrease the study power), by using a hierarchical model we were able to account for this variability and hence assess the independent effect of the treatment upon treatment outcomes. In contrast to our previous IPD analysis, where patients taking any other G5Ds were excluded as controls for patients taking a given G5D [2], in the present analyses we included individuals taking other G5Ds as control subjects. This approach is robust, given that the patients given other G5Ds are also likely to be more sick (i.e. to be failing on existing therapies). Removing all patients taking other G5Ds from our analyses may have removed the sickest individuals and introduced further bias. 
TABLE 4 Matching of covariates achieved using one-to-one propensity score matching, with replacement, for clofazimine (CFZ) versus no CFZI, within studies where CFZ was used

\begin{tabular}{|c|c|c|c|c|c|c|}
\hline & \multirow[t]{2}{*}{$\begin{array}{c}\text { Patients } \\
\text { taking CFZ }\end{array}$} & \multirow[t]{2}{*}{$\begin{array}{c}\text { Patients } \\
\text { not taking CFZ }\end{array}$} & \multicolumn{2}{|c|}{$\begin{array}{l}\text { Before matching } \\
\text { (CFZ versus no CFZ) }\end{array}$} & \multicolumn{2}{|c|}{$\begin{array}{l}\text { After matching } \\
\text { (CFZ versus no CFZ) }\end{array}$} \\
\hline & & & p-value & $\begin{array}{l}\text { Standardised } \\
\text { difference }{ }^{\#} \%\end{array}$ & p-value & $\begin{array}{l}\text { Standardised } \\
\text { difference }{ }^{\#} \%\end{array}$ \\
\hline Male & $0.62 \pm 0.49$ & $0.65 \pm 0.48$ & 0.162 & -6.7 & 0.5102 & 1.3 \\
\hline Age years & $34.28 \pm 12.51$ & $38.06 \pm 14.52$ & $<0.0001$ & 9.85 & 0.207 & 2.4 \\
\hline Extensive disease & $0.79 \pm 0.4$ & $0.79 \pm 0.41$ & 0.724 & 1.9 & 0.0014 & 5.9 \\
\hline HIV infection & $0.1 \pm 0.3$ & $0.36 \pm 0.48$ & $<0.0001$ & -64.5 & 0.1066 & 2.7 \\
\hline Antibiotics used in the intensive phase & $6.15 \pm 1.39$ & $4.4 \pm 2.29$ & $<0.0001$ & -32.6 & 0.053 & -2.3 \\
\hline Total therapy months & $22.53 \pm 11.44$ & $16.6 \pm 12.32$ & $<0.0001$ & -17.6 & 0.054 & 3.5 \\
\hline Logit of PS & $0.13 \pm 0.99$ & $-1.22 \pm 1.35$ & $<0.0001$ & -40.7 & 0.039 & -0.1 \\
\hline
\end{tabular}

This table is presented as an example. This matching was performed for all group 5 drugs, for which these comparisons were performed. TB: tuberculosis; MDR-TB: multidrug-resistant TB; PS: propensity score. \#: percentage mean difference as a percentage of the average standard deviation.

An important study limitation was the observational nature of the included cohort data. Since G5Ds have uncertain efficacy, and well-known toxicity, guidelines recommend that G5Ds should be reserved for the patients with the fewest effective treatment options [3]. This can be seen most clearly in the low rate of treatment success treated with any G5D, despite adjustment. The lower treatment success seen in the multivariable analyses probably indicates an effect of treatment allocation to more severely ill patients, rather than an adverse biological effect.

\section{Conclusions}

This individual patient data meta-analysis found that G5Ds had limited benefit in treating patients with MDR-TB. The recent availability of bedaquiline [35] and delamanid [36] has provided some new hope for patients with advanced drug resistance. Given that regimens for MDR-TB remain toxic, lengthy and complex, new drug development remains an urgent priority in global TB control.

TABLE 5 The relationship between drug use and treatment success compared to death, failure or relapse, using multivariableand propensity score-matched analyses to adjust for potential confounding ${ }^{\#}$

\begin{tabular}{|c|c|c|c|c|}
\hline & $\begin{array}{l}\text { Unadjusted } \\
\text { estimate }\end{array}$ & $\begin{array}{c}\text { Adjusted multivariable } \\
\text { estimate }\end{array}$ & $\begin{array}{l}\text { Adjusted estimate } \\
\text { using matching }\end{array}$ & $\begin{array}{l}\text { Measures of heterogeneity } \\
\text { with matched model } I_{R}^{2}\end{array}$ \\
\hline \multicolumn{5}{|c|}{$\begin{array}{l}\text { G5D used (comparison is with NG5a } \\
\text { patients from same centres) }\end{array}$} \\
\hline Clofazimine & $0.4(0.3-0.6)$ & $0.4(0.3-0.6)$ & $0.6(0.2-1.6)$ & 0.445 \\
\hline Amoxicillin/clavulanic acid & $0.6(0.3-0.9)$ & $0.4(0.3-0.5)$ & $0.9(0.4-1.9)$ & 0.337 \\
\hline Thioacetazone & $0.9(0.6-1.2)$ & $0.8(0.5-1.1)$ & $0.8(0.5-1.2)^{+}$ & NA \\
\hline Clofazimine & $1.0(0.4-2.6)$ & $1.1(0.4-2.8)$ & $1.4(0.5-4.0)$ & 0.444 \\
\hline Amoxicillin/clavulanic acid & $0.4(0.3-0.6)$ & $0.4(0.2-0.8)$ & $0.9(0.4-1.9)$ & 0.303 \\
\hline Thioacetazone & $1.8(0.6-5.1)^{+}$ & $2.1(0.8-5.6)$ & $2.1(0.8-5.5)$ & 0.447 \\
\hline Clarithromycin & $0.7(0.4-1.5)$ & $0.7(0.3-1.3)$ & $0.7(0.2-1.9)$ & 0.310 \\
\hline \multicolumn{5}{|c|}{$\begin{array}{l}\text { Data are presented as OR }(95 \% \mathrm{Cl}) \text {, unless otherwise stated. The } I_{R}^{2} \text { statistic quantifies the between-study variability. G5D: group } 5 \text { drug; NG5a } \\
\text { patients from studies where that G5D was available, but who did not receive any G5Ds; NG5b: unexposed patients in studies where that G5D } \\
\text { was not available; NA: not applicable, as only fixed-effects model used. \#: Linezolid and terizidone were not included in these adjusted } \\
\text { analyses, as too few patients received these drugs; }{ }^{\uparrow} \text { : adjusting for age, sex, extent of tuberculosis (TB), prior TB, prior multidrug-resistant TB } \\
\text { number of drugs in the intensive phase, total duration of therapy and HIV status. Analyses include subjects within studies where at least one } \\
\text { person used the G5D; }{ }^{+} \text {: hierarchical model for thioacetazone did not converge on account of substantial heterogeneity between studies, hence } \\
\text { results from fixed-effects model are presented. }\end{array}$} \\
\hline
\end{tabular}


TABLE 6 The relationship between drug use and treatment success compared to death, failure, relapse or loss to follow-up, using multivariable- and propensity score-matched analyses to adjust for potential confounding ${ }^{\#}$

Unadjusted estimate

Adjusted multivariable estimate ${ }^{\pi}$

\section{Adjusted estimate using matching}

Measures of heterogeneity with matched model $I_{R}^{2}$

\section{G5D used (comparison is with NG5a patients from same centres)}

Clofazimine

Amoxicillin/clavulanic acid

Thioacetazone

Clarithromycin

\section{G5D used (comparison is with NG5b}

patients from other centres)

Clofazimine

Amoxicillin/clavulanic acid

Thioacetazone

Clarithromycin

\section{6}

0.7

0.9

0.7

1.1

0.8

2.4

0.8

$(0.4-0.7)$
$(0.6-0.9)$
$(0.7-1.2)$
$(0.6-0.8)$

(0.6-0.8)

(0.5-2.3)

(0.4-1.3)

(0.9-6.4)

(0.4-1.5)

\author{
0.5 \\ 0.6 \\ 0.9 \\ 0.7
}

0.8

0.4

2.8

0.7
$(0.4-0.7)$
$(0.4-0.7)$
$(0.6-1.3)$
$(0.6-0.9)$

(0.4-1.9)

(0.2-0.8)

$(1.0-7.3)^{\S}$

(0.4-1.4)
0.7

0.9

0.9

0.7

(0.3-1.4

(0.5-1.5)

$(0.7-1.3)^{+}$

(0.4-1.0)

0.424

0.392

NA

0.138

Data are presented as OR (95\% CI), unless otherwise stated. The $I_{R}^{2}$ statistic quantifies the between-study variability. G5D: group 5 drug; NG5a: patients from studies where that G5D was available, but who did not receive any G5Ds; NG5b: unexposed patients in studies where that G5D was not available; NA: not applicable, as only fixed-effects model used. \#: Linezolid and terizidone were not included in these adjusted analyses, as too few patients received these drugs; ${ }^{\text {": }}$ adjusting for age, sex, extent of tuberculosis (TB), prior TB, prior multidrug-resistant TB, number of drugs in the intensive phase, total duration of therapy and HIV status. Analyses include subjects within studies where at least one person used the $\mathrm{GSD}^{+}{ }^{+}$: hierarchical model did not converge on account of substantial heterogeneity between studies, hence results from fixed-effects model are presented; ${ }^{\S}: p=0.043$.

\section{Acknowledgements}

Members of the Collaborative Group for Meta-Analysis of Individual Patient Data in MDR-TB are as follows: S. Ahuja (Bureau of Tuberculosis, New York, NY, USA); D. Ashkin (A.G. Holley Hospital, Lantana, FL, USA); M. Avendaño (University of Toronto, Toronto, Canada); R. Banerjee (Mayo Clinic, Rochester, MN, USA); M. Bauer, M.G. Hollm-Delgado, M. Pai and L. Shah (Montreal Chest Institute, McGill University, Montreal, Canada); J. N. Bayona (The Dartmouth Center for Health Care Delivery Science, Hanover, NH, USA); M. Becerra (Harvard Medical School, Boston, MA, USA and Partners in Health, Boston); M. Burgos (University of New Mexico School of Medicine, Albuquerque, NM, USA); R. Centis, L. D'Ambrosio and G.B. Migliori (World Health Organization Collaborating Centre for TB and Lung Diseases, Care and Research Institute, Fondazione S. Maugeri, Tradate, Italy); E.D. Chan (Denver Veterans Affair Medical Center, Denver, CO, USA); C.Y. Chiang (Wan Fang Hospital, School of Medicine-Taipei Medical University, Taiwan); W.C.M. de Lange, R. van Altena and T.S. van der Werf (University Medical Center Groningen, Groningen, The Netherlands); K. DeRiemer (UC Davis School of Medicine, Davis, CA, USA); N.H. Dung (Ho Chi Minh City, Viet Nam); D. Enarson (International Union against Tuberculosis and Lung Disease, Paris, France); D. Falzon and R.M. Granich (World Health Organization, Geneva, Switzerland); K. Flanagan (MRC Laboratories, Banjul, The Gambia); J. Flood (California Department of Public Health, Sacramento, CA, USA); N. Gandhi (Albert Einstein College of Medicine, Bronx, NY, USA); L. Garcia-Garcia (Instituto Nacional de Salud Publica, Mexico, Mexico); T.H. Holtz (Thailand MOPH \& US CDC Collaboration, Bangkok, Thailand); M. Iseman and M.J. Strand (National Jewish Health, Denver); L.G. Jarlsberg and S. Royce (University of California, San Francisco, San Francisco, CA, USA); S. Keshavjee, H.R. Kim and C.D. Mitnick (Harvard Medical School, Boston); J. Lancaster and M. van der Walt (South African Medical Research Council, Pretoria, South Africa); C. Lange (Medical Clinic, Tuberculosis Center Borstel, Borstel, Germany); V. Leimane and V. Riekstina (Clinic of Tuberculosis and Lung Diseases, Riga, Latvia); C.C. Leung (Tuberculosis and Chest Services, Hong Kong); J. Li (New York City Health and Mental Hygiene, New York); M. Narita (University of Washington, Seattle, WA, USA); P. O'Riordan (City Road Medical Centre, London, UK); D. Palmero (Hospital F.J. Muniz, Buenos Aires, Argentina); S.K. Park (TB Center, Seoul, Korea); J. Pena (Universidad Autonoma Madrid, Madrid, Spain); C. Pérez-Guzmán (Instituto de Salud del Estado de Aguascalientes, Mexico); A. Ponce-de-Leon and J. Sifuentes-Osornio (Instituto Nacional de Ciencias Medicas y de Nutricion "Salvador Zubiran", Mexico, Mexico); M.I.D. Quelapio and T.E. Tupasi (Tropical Disease Foundation, Makati City, Philippines); J. Robert (Bacteriologie-Hygiene - UPMC, Paris); H.S. Schaaf (Desmond Tutu TB Centre, Department of Paediatrics and Child Health, Faculty of Health Sciences, Stellenbosch University, Tygerberg, South Africa); K.J. Seung and S.S. Shin (Brigham and Women's Hospital, Boston); T.S. Shim (University of Ulsan College of Medicine, Seoul, Korea); Y. Shiraishi (Fukujuji Hospital, Tokyo, Japan; G. Sotgiu (University of Sassari, Sassari, Italy); P. Tabarsi (Shaheed Beheshti Medical University, Tehran, Iran); M.H. Vargas (Instituto Nacional de Enfermedades Respiratorias, Mexico, Mexico); J. Westenhouse (Center for Infectious Diseases-California Department of Public Health, Sacramento, CA, USA); W.W. Yew (Grantham Hospital, Hong Kong); and J.J. Yim (Seoul National University College of Medicine, Seoul, Korea).

\section{References}

1 World Health Organization (WHO). Global Tuberculosis Report 2015. Geneva, WHO, 2015.

2 Ahuja SD, Ashkin D, Avendano M, et al. Multidrug resistant pulmonary tuberculosis treatment regimens and patient outcomes: an individual patient data meta-analysis of 9,153 patients. PLoS Med 2012; 9: e1001300.

3 World Health Organization (WHO). Guidelines for the Programmatic Management of Drug-Resistant Tuberculosis. Geneva, WHO, 2011. 
4 Dooley KE, Obuku EA, Durakovic N, et al. World Health Organization group 5 drugs for the treatment of drug-resistant tuberculosis: unclear efficacy or untapped potential? J Infect Dis 2013; 207: 1352-1358.

5 Chang KC, Yew WW, Tam CM, et al. WHO group 5 drugs and difficult multidrug-resistant tuberculosis: a systematic review with cohort analysis and meta-analysis. Antimicrob Agents Chemother 2013; 57: 4097-4104.

6 Winters N, Butler-Laporte G, Menzies D. Efficacy and safety of World Health Organization group 5 drugs for multidrug-resistant tuberculosis treatment. Eur Respir J 2015; 46: 1461-1470.

7 Tyagi S, Ammerman NC, Li S, et al. Clofazimine shortens the duration of the first-line treatment regimen for experimental chemotherapy of tuberculosis. Proc Natl Acad Sci USA 2015; 112: 869-874.

8 Lee M, Lee J, Carroll MW, et al. Linezolid for treatment of chronic extensively drug-resistant tuberculosis. $N$ Engl J Med 2012; 367: 1508-1518.

9 Tang S, Yao L, Hao X, et al. Efficacy, safety and tolerability of linezolid for the treatment of XDR-TB: a study in China. Eur Respir J 2015; 45: 161-170.

10 Tang S, Yao L, Hao X, et al. Clofazimine for the treatment of multidrug-resistant tuberculosis: prospective, multicenter, randomized controlled study in China. Clin Infect Dis 2015; 60: 1361-1367.

11 Dey $\mathrm{T}$, Brigden $\mathrm{G}$, Cox $\mathrm{H}$, et al. Outcomes of clofazimine for the treatment of drug-resistant tuberculosis: a systematic review and meta-analysis. J Antimicrob Chemother 2013; 68: 284-293.

12 Gopal M, Padayatchi N, Metcalfe JZ, et al. Systematic review of clofazimine for the treatment of drug-resistant tuberculosis. Int J Tuberc Lung Dis 2013; 17: 1001-1007.

13 Stewart LA, Tierney JF. To IPD or not to IPD? Advantages and disadvantages of systematic reviews using individual patient data. Eval Health Prof 2002; 25: 76-97.

14 Simmonds MC, Higgins JP. Covariate heterogeneity in meta-analysis: criteria for deciding between metaregression and individual patient data. Stat Med 2007; 26: 2982-2999.

15 Ackçakir Y. Correlates of Treatment Outcomes of Multidrug-Resistant Tuberculosis (MDR-TB): a Systematic Review and Meta-Analysis. Montreal, McGill University; 2010. http://digitool.library.mcgill.ca/R/-?func=dbinjump-full\&object_id=86914 Date last accessed: August 6, 2016. Date last updated: 2010.

16 Johnston JC, Shahidi NC, Sadatsafavi M, et al. Treatment outcomes of multidrug-resistant tuberculosis: a systematic review and meta-analysis. PloS One 2009; 4: e6914.

17 Orenstein EW, Basu S, Shah NS, et al. Treatment outcomes among patients with multidrug-resistant tuberculosis: systematic review and meta-analysis. Lancet Infect Dis 2009; 9: 153-161.

18 Van Deun A, Salim MA, Das AP, et al. Results of a standardised regimen for multidrug-resistant tuberculosis in Bangladesh. Int J Tuberc Lung Dis 2004; 8: 560-567.

19 Laserson KF, Thorpe LE, Leimane V, et al. Speaking the same language: treatment outcome definitions for multidrug-resistant tuberculosis. Int I Tuberc Lung Dis 2005; 9: 640-645.

20 Bastos ML, Hussain H, Weyer K, et al. Treatment outcomes of patients with multidrug-resistant and extensively drug-resistant tuberculosis according to drug susceptibility testing to first- and second-line drugs: an individual patient data meta-analysis. Clin Infect Dis 2014; 59: 1364-1374.

21 Higgins JP, Thompson SG, Deeks JJ, et al. Measuring inconsistency in meta-analyses. BMJ 2003; 327: 557-560.

22 Fox GJ, Benedetti A, Mitnick CD, et al. Propensity score-based approaches to confounding by indication in individual patient data meta-analysis: non-standardized treatment for multidrug resistant tuberculosis. PloS One 2016; 11: e0151724.

23 Rosenbaum P, Rubin D. The central role of the propensity score in observational studies for causal effects. Biometrika 1983; 70: 41-55.

24 Coca-Perraillon M, ed. Matching with Propensity Scores to Reduce Bias in Observational Studies. Proceedings of the NorthEast SAS Users Group Conference (NESUG), Philadelphia, PA; 2006.

25 Austin PC. An introduction to propensity score methods for reducing the effects of confounding in observational studies. Multivariate Behav Res 2011; 46: 399-424.

26 Austin PC. The performance of different propensity score methods for estimating marginal hazard ratios. Stat Med 2013; 32: 2837-2849.

27 D'Agostino RB Jr. Propensity score methods for bias reduction in the comparison of a treatment to a nonrandomized control group. Stat Med 1998; 17: 2265-2281.

28 Normand ST, Landrum MB, Guadagnoli E, et al. Validating recommendations for coronary angiography following acute myocardial infarction in the elderly: a matched analysis using propensity scores. J Clin Epidemiol 2001; 54: $387-398$.

29 Jackson D, White IR, Riley RD. Quantifying the impact of between-study heterogeneity in multivariate meta-analyses. Stat Med 2012; 31: 3805-3820.

30 Stewart LA, Clarke M, Rovers M, et al. Preferred Reporting Items for Systematic Review and Meta-Analyses of individual participant data: the PRISMA-IPD statement. JAMA 2015; 313: 1657-1665.

31 DerSimonian R, Laird N. Meta-analysis in clinical trials. Control Clin Trials 1986; 7: 177-188.

32 Leimane V, Riekstina V, Holtz TH, et al. Clinical outcome of individualised treatment of multidrug-resistant tuberculosis in Latvia: a retrospective cohort study. Lancet 2005; 365: 318-326.

33 World Health Organization (WHO). WHO Treatment Guidelines for Drug-Resistant Tuberculosis: 2016 Update. Geneva, WHO, 2016.

34 Padayatchi N, Gopal M, Naidoo R, et al. Clofazimine in the treatment of extensively drug-resistant tuberculosis with HIV coinfection in South Africa: a retrospective cohort study. J Antimicrob Chemother 2014; 69: 3103-3107.

35 Diacon AH, Donald PR, Pym A, et al. Randomized pilot trial of eight weeks of bedaquiline (TMC207) treatment for multidrug-resistant tuberculosis: long-term outcome, tolerability, and effect on emergence of drug resistance. Antimicrob Agents Chemother 2012; 56: 3271-3276.

36 Gler MT, Skripconoka V, Sanchez-Garavito E, et al. Delamanid for multidrug-resistant pulmonary tuberculosis. N Engl J Med 2012; 366: 2151-2160. 